\title{
Effect of Outlier Elimination on the 99th Percentile Upper Reference Limits of High-Sensitivity Cardiac Troponin I Assays Based on a Strictly Selected Healthy Reference Population
}

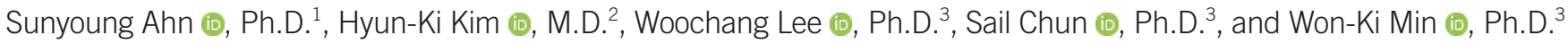 \\ ${ }^{1}$ Department of Laboratory Medicine, Yonsei University College of Medicine, Severance Hospital, Seoul, Korea; ${ }^{2}$ Department of Laboratory Medicine, \\ University of Ulsan College of Medicine, Ulsan University Hospital, Ulsan, Korea; ${ }^{3}$ Department of Laboratory Medicine, University of Ulsan College of \\ Medicine, Asan Medical Center, Seoul, Korea
}

Background: We established high-sensitivity cardiac troponin I (hsTnl) 99th percentile upper reference limits (URLS) for the Centaur XPT High-Sensitivity Troponin I assay (Centaur hsTnl; Siemens, Erlangen, Germany) and Atellica IM High-Sensitivity Troponin I assay (Atellica hsTnl; Siemens) and assessed the effect of outlier elimination.

Methods: The reference population comprised 380 men and 387 women, satisfying the strict systematic reference population criteria. After reference population verification by the N-terminal pro-B-type natriuretic peptide (NT-proBNP) assay, 99th percentile URLs for Centaur hsTnl and Atellica hsTnl were calculated before and after outlier elimination.

Results: The 99th percentile URL for Centaur hsTnl was 60.4 (men, 74.7; women, 57.5) $\mathrm{ng} / \mathrm{L}$ and that for Atellica hsTnl was 59.6 (men, 75.2; women, 55.1) ng/L. After the elimination of 61 (8.0\%) outlier samples in Centaur hsTnl and 58 (7.6\%) in Atellica hsTnl, the 99th percentile URLs were $13.5 \mathrm{ng} / \mathrm{L}$ (men, $15.3 \mathrm{ng} / \mathrm{L}$; women, $11.9 \mathrm{ng} / \mathrm{L}$ ) and $13.4 \mathrm{ng} / \mathrm{L}$ (men, $15.5 \mathrm{ng} / \mathrm{L}$; women, $12.9 \mathrm{ng} / \mathrm{L}$ ), respectively, significantly lower than those before outlier elimination. The CVs at the 99th percentile URLs were $5.2 \%$ and $3.5 \%$, respectively. The measurable fractions among the reference population were $91.5 \%$ and $93.4 \%$, respectively. Performance evaluation of Atellica B-type natriuretic peptide (BNP), Atellica NT-proBNP, Centaur hsTnl, and Atellica hsTnl showed outstanding results.

Conclusions: The Korean hsTnl 99th percentile URLs calculated in this study were significantly lower after outlier elimination than before. Centaur hsTnl and Atellica hsTnl meet the "Guideline acceptable" and "Level 3 (second generation, high sensitivity)" requirements, satisfying international standards.

Key Words: Ninety-ninth percentile, Upper reference limit, Korean, Outlier, ADVIA Centaur XPT High-Sensitivity Troponin I assay, Atellica IM High-Sensitivity Troponin I assay
Received: February 21, 2021

Revision received: June 13, 2021

Accepted: November 30, 2021

\section{Corresponding author:}

Won-Ki Min, M.D., Ph.D.

Department of Laboratory Medicine,

University of Ulsan College of Medicine,

Asan Medical Center, 88 Olympic-ro 43-gil,

Songpa-gu, Seoul 03722, Korea

Tel.: +82-2-3610-4503

Fax: +82-2-478-0884.

E-mail:wkmin@amc.seoul.kr

\section{INTRODUCTION}

Troponin (Tn), a structural protein, is a component of actin-myosin fibers and is involved in calcium-mediated muscle contraction. Among Tns, cardiac (c)TnT and cTnl are often used to di- agnose and monitor cardiac diseases as they are specific to cardiac muscles [1, 2]. According to the European Society of Cardiology, American College of Cardiology, American Heart Association, and World Heart Federation Task Force Fourth Universal Definitions of Myocardial Injury and Myocardial Infarction, 
myocardial infarction can be diagnosed when cTn values are above the 99th percentile of the upper reference limit (URL) values determined in healthy individuals and when at least one cardiac symptom specified in the guidelines occurs [2]. Hence, for rapid and accurate diagnosis of myocardial infarction, it is crucial to measure cTn values accurately and to establish a reference range for cTn based on a strictly selected healthy reference population [3, 4].

Multiple studies have reported that the 99th percentile URLS used as a cTn reference range in diagnosing myocardial infarction vary across demographics, especially considering the size and clinical characteristics of the reference population [5-9]. In 2014, Sandoval and Apple [10] reported strict standards for selecting a reference population to establish 99th percentile URLS for cTn. According to these standards, at least 300 men and 300 women should be recruited. The American Association for Clinical Chemistry (AACC) and International Federation of Clinical Chemistry and Laboratory Medicine (IFCC) guidelines suggest the same criteria for the reference population size required to define the 99th percentile URL for each sex [11]. However, Hickman, et al. [12] showed that outlier elimination can dramatically alter cTn 99th percentile URLs. Thus, incorrect outlier evaluation may significantly increase 99th percentile URLs [6, 9 , $12,13]$.

Recently, the ADVIA Centaur High-Sensitivity Troponin I assay (Centaur hsTnl) (Siemens, Erlangen, Germany), which is a fully automated version of the Centaur XP Tnl-Ultra assay (Centaur Tnl; Siemens), and the Atellica IM High-Sensitivity Troponin I assay (Atellica hsTnl; Siemens) were introduced in clinical laboratories. The Centaur Tnl and both hsTnl assays are chemiluminescence immunoassays. However, the hsTnl assays newly employ trisulfopropyl acridinium ester, a strong anionic label with very low non-specific binding and the same high light yield that confers the hsTnl assays a 10-fold improvement in low-end precision sensitivity. Additionally, the hsTnl assays are robust, with a high tolerance for common interferents, such as biotin, hemolysis, and alkaline phosphatase. Further, they show an optimal precision of $>90 \%$ across a measurable range. The two hsTnl assays have subtle differences. The time to first result is 18 and 10 minutes for Centaur hsTnl and Atellica hsTnl, respectively. In addition, the 99th percentile value (for men and women combined and for serum, according to the package insert) is $46.8 \mathrm{ng} / \mathrm{L}$ and $45.4 \mathrm{ng} / \mathrm{L}$ for Centaur hsTnl and Atellica hsTnl, respectively. Both assays analyze the full cardiac panel, including high-sensitivity Tnl (hsTnl), B-type natriuretic peptide (BNP), N-terminal pro-brain BNP (NT-proBNP), myoglobulin, and creatine kinase myocardial band, in a single platform.

We calculated 99th percentile URLs for cTnl for Centaur hsTnl and Atellica hsTnl in a healthy Korean population of sufficient size, meeting the selection criteria suggested by Sandoval and Apple and the AACC and IFCC guidelines [10, 11], to determine the effect of outlier elimination on the 99th percentile URLs. Additionally, we evaluated the performance of Centaur hsTnl, Atellica hsTnl, Atellica IM BNP, and Atellica IM NTproBNP, all of which can be used to assess cardiac function.

\section{MATERIALS AND METHODS}

\section{Ethics considerations}

This retrospective observational study was reviewed and approved by the Institutional Review Board (IRB) of Asan Medical Center, Seoul, Korea (approval number.: 2018-0731). As we used residual serum samples that had been used for regular laboratory assays and were completely anonymized, the requirement for informed consent was waived by the IRB.

\section{Reference population selection and sample preparation}

Residual serum samples bottled in BD Vacutainer Plus Plastic Serum Tubes (BD, Franklin Lakes, NJ, USA) from subjects who visited Asan Medical Center for general health checkups between October 10, 2019 and February 25, 2020 were collected. Within 24 hours after collection, the samples were divided into four 250- $\mu \mathrm{L}$ aliquots in Eppendorf tubes (Eppendorf North America, Enfield, CT, USA) and frozen at $-70^{\circ} \mathrm{C}$ until assaying. At the end of the sample collection, all frozen samples were thawed, vortexed, and centrifuged for analysis. These samples were used to evaluate cTnl 99th percentile URLs. The inclusion criteria for reference population selection and the study flowchart are presented in Fig. 1.

To assess "clinical history for known cardiovascular disease and medication usage," we reviewed the subjects' checkup questionnaire data, electronic medical records, and laboratory test results at the time of recruitment to screen those with active or historical cardiac diseases, including acute coronary syndromes, congestive heart failure, myocarditis, ventricular tachyarrhythmias, and percutaneous coronary intervention or cardiac medication use. All subjects also underwent NT-proBNP measurement, which is a criterion for selecting the reference population, to screen out those with current or historical asymptomatic cardiac diseases, as these can cause outliers and may affect the calculation of the 99th percentile URL [14, 15].

The NT-proBNP cutoff was set at the age- and sex-specific 
Ahn S, et al.

The 99th percentile URL of hsTnl assays
ANNALS OF

LABORATORY MEDICINE

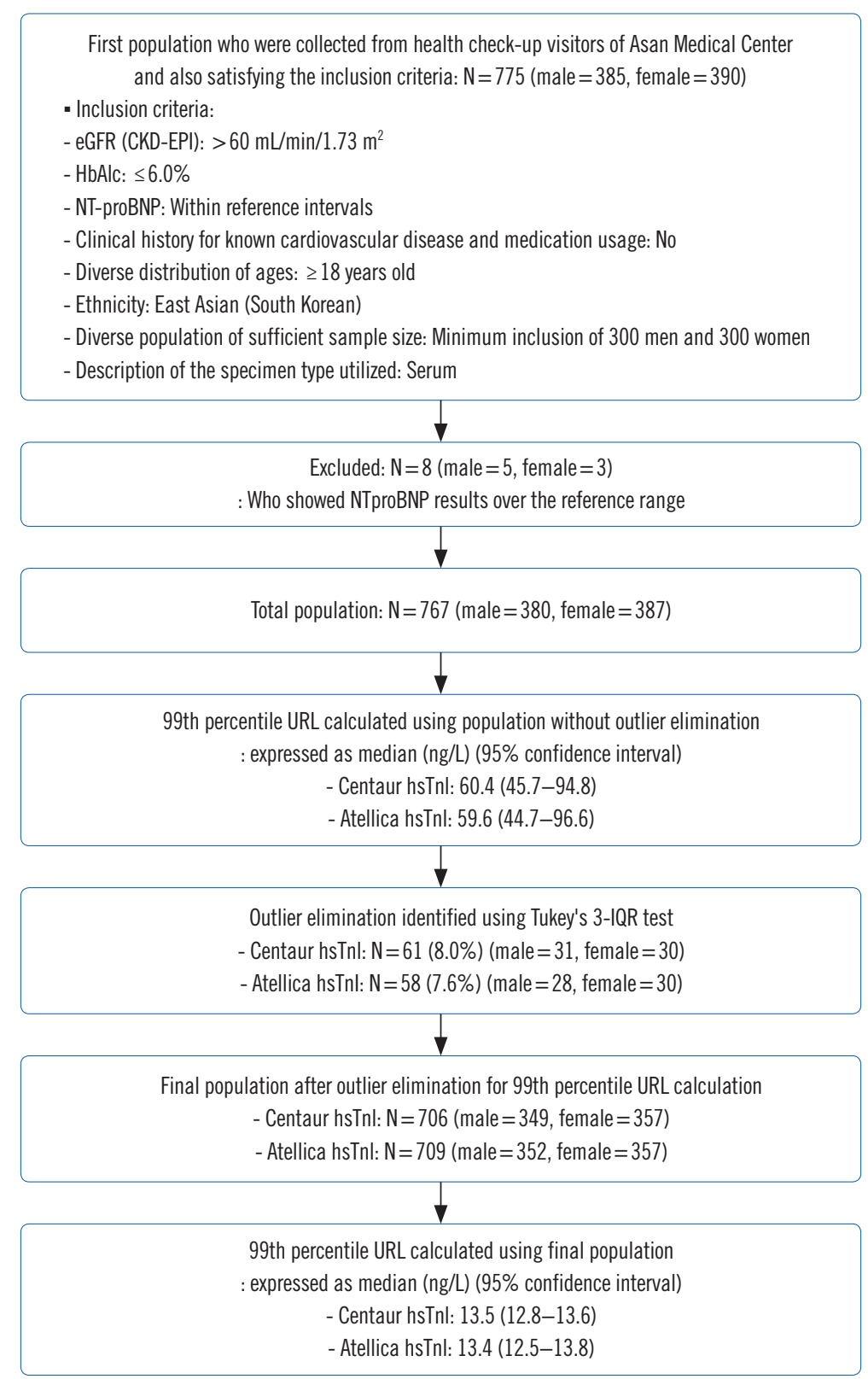

Fig. 1. Flow diagram showing the reference population used, process of outlier elimination, and the final 99th percentile URLs calculated for Centaur hsTnl and Atellica hsTnl.

Abbreviations: eGFR, estimated glomerular filtration rate; CKD-EPI, Chronic Kidney Disease Epidemiology Collaboration; HbA1c, hemoglobin Alc; NT-proBNP, N-terminal pro-B-type natriuretic peptide; URL, upper reference limit; hsTnl, high-sensitivity troponin I; IQR, interquartile range.

values provided by the manufacturer (package insert of the Elecsys NT-proBNP assay, Roche, Basel, Switzerland) [18-54 years: 143.0 (men) or 208.4 (women) ng/L; 55-64 years: 650.8 (men) or 912.2 (women) ng/L; 65-74 years: 512.0 (men) or 533.4 (women) ng/L; $\geq 75$ years: 603.6 (men) or 665.0 (women) $\mathrm{ng} / \mathrm{L}$ ]. To include at least 300 men and 300 women in the calculation of the cTnl 99th percentile URL, the subjects were divided into the following age groups: <30, 30-39, 40-49, $50-$
$59,60-69$, and $\geq 70$ years. The subjects were further divided into subgroups based on age $\leq 55$ years or $>55$ years to compare the effect of age on Tnl 99th percentile URLs.

\section{Evaluation of precision}

Precision was evaluated according to the CLSI guidelines EP5A3 [16]. Low, medium, and high levels of quality control (QC) materials (Liquichek Cardiac Markers Plus Control, lot numbers 
99562, 99563, and 99565; Bio-Rad, Hercules, CA, USA) were used for Atellica IM BNP, Atellica IM NT-pro BNP, Centaur hsTnl, and Atellica hsTnl. Each QC standard was analyzed in duplicate and twice a day (with a $\geq 2$-hour interval between analyses) for 20 days.

\section{Evaluation of linearity}

Linearity was evaluated according to the CLSI guidelines EP6-A [17], using Atellica IM BNP Master Curve Material, Atellica IM PBNP Master Curve Material, Atellica IM TNIH Master Curve Material, and ADVIA Centaur TNIH Master Curve Material (all from Siemens). Each material was assayed four times. The data were subjected to polynomial regression analysis, and the deviation from linearity was calculated. The allowable nonlinearity was set at $27.9 \%$ for hsTnl and $13 \%$ for BNP and ProBNP based on the total allowable error of the Desirable Biological Variation Database specifications available on the Westgard website [18].

\section{Method comparison}

Method comparison was conducted according to the CLSI guidelines EP9-A2 [19]. For BNP and hsTnl, Centaur XPT performance was compared with that of Atellica IM. For NTproBNP, Cobas C8000 (Roche) was used in comparison with Atellica IM. To compare cTnl and hsTnl, Centaur XPT was used. Forty residual serum samples were analyzed separately for each of the four analytes. Data were analyzed using Deming regression, and the slope, intercept, and correlation coefficients ( $R$ values) were calculated. $\mathrm{R}^{2} \geq 0.95$ indicated a linear correlation between the two reagents over the comparison experiment range.

\section{Determination of CVs at the 99th percentile URLs}

We obtained whole blood samples from two healthy volunteers with informed consent. Serum was separated immediately after blood collection and stored at $-70^{\circ} \mathrm{C}$. The serum samples were thawed right before use and spiked with residual serum samples that showed high cTnl values to prepare serum pools with $\mathrm{Tnl}$ values of 26.5-106.3 $\mathrm{ng} / \mathrm{L}$. Using the multilevel pools, we generated precision profile plots. Samples with six Tnl values spanning the 99th percentile URLs were prepared. The Tnl value of each sample was measured 80 times using Centaur hsTnl and Atellica hsTnl. The CVs at each Tnl value for each assay were then analyzed according to the CLSI guidelines EP5A3 [16]. Based on the CVs of the six serum pools, we generated precision profile plots using the Variance Function Program (version 15.0; W. A. Sadler, Christchurch, New Zealand) and calculated the CVs at the 99th percentile URLs for Centaur hsTnl and Atellica hsTnl.

\section{Statistical analysis}

The presence of outliers in data is a major problem and strongly affects 99th percentile URLs [8, 9, 11, 20, 21]. We determined the 99th percentile URLs using the total samples before and after outlier elimination by a one-tailed non-parametric method with 3,000 bootstrap replications and a 95\% confidence interval (CI) in SPSS (version 18.0.0; IBM, Armonk, NY, USA). Outliers were identified using Tukey's 3-interquartile range (IQR) test using the following formula: outlier hsTnl value $>Q 3+3 I Q R$, where $\mathrm{Q} 3$ and IQR are the third quartile and interquartile range (Q3-Q1) of the hsTnl distribution, respectively [9, 12]. We eliminated outliers manually. Since hsTnl values are higher in older subjects, especially at the reference age of 55 years in the general population, we stratified the reference population by age ( $\leq 55$ years and $>55$ years) and determined the 99th percentile URLs for each group $[8,9]$.

As hsTnl values were not normally distributed, sex- and agerelated differences were evaluated using the Mann-Whitney and Kruskal-Wallis tests. hsTnl values are represented as the median and 95\% Cl [22]. Statistical significance was set at $P<0.05$.

For general statistical analysis, including the four analyte performance evaluations, EP Evaluator Release 9 (Data Innovations, Colchester, VT, USA) and Microsoft Excel 2016 (Microsoft, Redmond, WA, USA) were used. To calculate the 99th percentile URLs and CVs, Variance Function Program version 15.0 and SPSS version 18.0.0. (IBM) were used.

\section{RESULTS}

\section{Precision}

The results of precision analyses are presented in Supplemental Data Table S1. For all assays, the total CV was 2.0\%-4.1\%. The CVs at the 99th percentile URLs were both $<10 \%$, which satisfied the criteria for hsTn assays according to the AACC and IFCC guidelines [11].

\section{Linearity}

The results of linearity evaluation are presented in Supplemental Data Fig. S1. Linearity was observed throughout the estimated analytical range for all analytes. $\mathrm{R}^{2} \geq 0.99$ was observed for all analytes, with $\mathrm{R}^{2}=0.99$ for Centaur hsTnl at 0.0-19,318.6 ng/L, $\mathrm{R}^{2}=0.99$ for Atellica hsTnl at 1.9-18,884.9 ng/L, R²=0.99 for Atellica IM 
Ahn S, et al.

The 99th percentile URL of hsTnl assays
ANNALS OF

LABORATORY MEDICINE
BNP at $0.9-4,000.1 \mathrm{ng} / \mathrm{L}$, and $\mathrm{R}^{2}=0.99$ for Atellica IM NTproBNP at 0.6-29,593.9 $\mathrm{ng} / \mathrm{L}$. The percentage recovery ranged from $90 \%$ (BNP, low value) to 101\% (NT-proBNP, high value).

\section{Method comparison}

Scatter and bias plots of the results of method comparison for BNP using Atellica IM and Centaur XPT, for NT-proBNP using Atellica IM and Roche Cobas 8000, for hsTnl using Atellica IM and Centaur XPT, and for cTnl and hsTnl using Centaur XPT for both are presented in Supplemental Data Fig. S2. For all analytes, the results showed strong correlations $\left(R^{2} \geq 0.95\right)$. Specifically, the correlation equation for BNP between Atellica IM and Centaur XPT was $y=0.98 x+0.69\left(R^{2}=0.99\right)$, showing a strong correlation between the two assays. The correlation coefficient for NT-proBNP between Atellica IM and Roche Cobas 8000 was strong $\left(R^{2}=0.99\right)$, with a correlation equation of $y=1.22$ $x+23.35$. The correlation equation and coefficient for hsTnl between Atellica hsTnl and Centaur hsTnl were strong ( $y=0.99$ $\mathrm{x}$-11.99, $\mathrm{R}^{2}=0.99$ ). cTnl values using Centaur XPT and hsTnl values using Centaur XPT showed strong correlation $\left(R^{2}=0.96\right)$. The correlation equation slope was 0.853 , indicating a proportional difference between the two assays. Hence, it is essential for institutions to re-establish 99th percentile URLs when replacing Centaur Tnl with Centaur hsTnl.

The 99th percentile reference values for hsTnl assays

After considering the NT-proBNP assay results, five men and three women showed higher values than the aforementioned cutoff values and were excluded from the reference population. We obtained 380 samples from men and 387 samples from

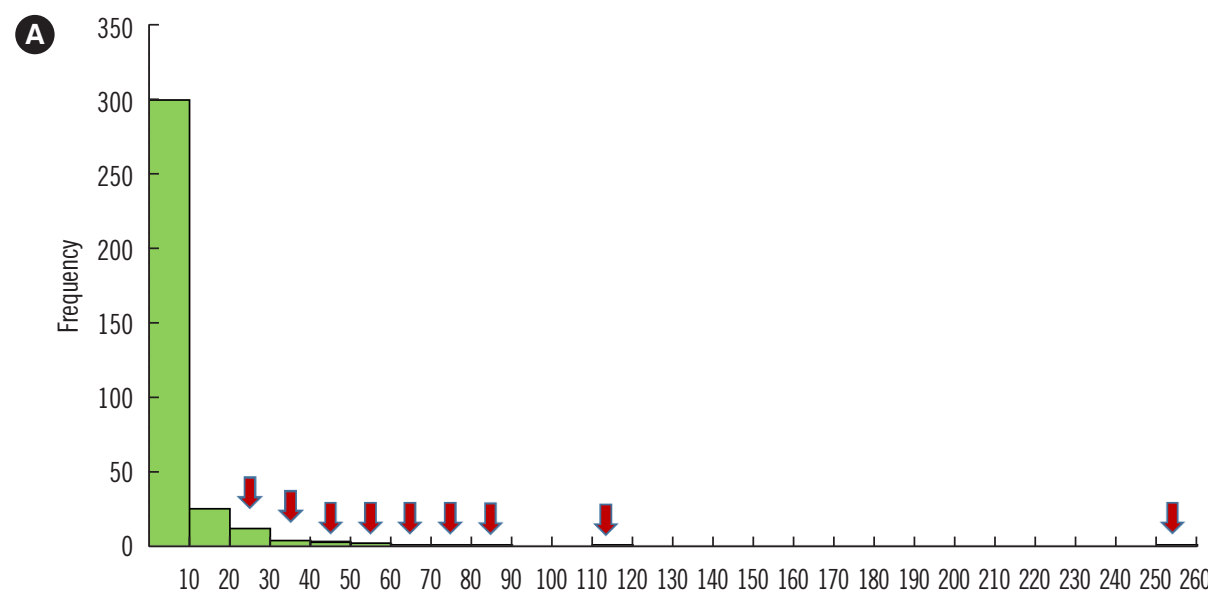

$\mathrm{Tnl}(\mathrm{ng} / \mathrm{L})$

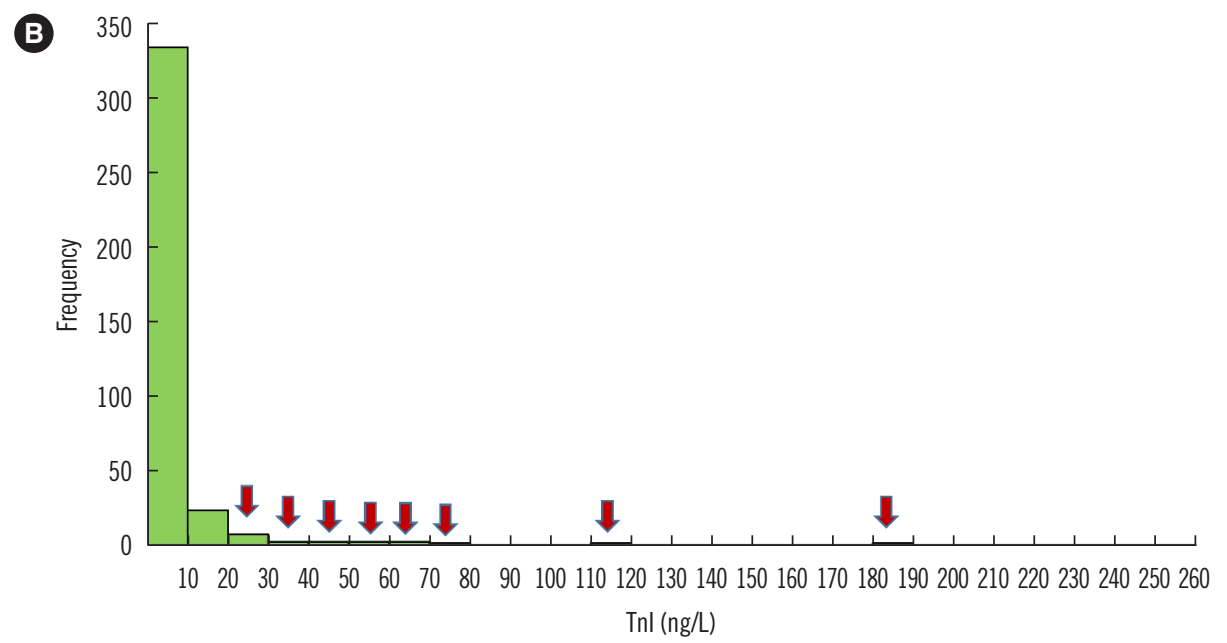

Fig. 2. Distribution of Tnl values in the reference population, which was selected according to the strict selection criteria suggested by Sandoval, et al. [10], without outlier elimination. Arrows indicate subjects with outlying Tnl values according to Tukey's 3-IQR method. (A) Men, (B) women.

Abbreviations: Tnl, troponin I; IQR, interquartile range. 
women before outlier elimination. Fig. 2 shows the $\mathrm{Tnl}$ value distribution in the reference population in men and women before outlier elimination.

The hsTnl value distributions according to age and sex in the reference population are shown in Figs. 3 and 4. Overall, hsTnl values were higher in men than in women within the same age group and tended to increase with age.

The 99th percentile URLs are listed in Table 1. The 99th percentile URLs of Centaur hsTnl and Atellica hsTnl before outlier elimination were 60.4 and $59.6 \mathrm{ng} / \mathrm{L}$, respectively. After outlier elimination, which accounted for $6.6 \%-10.1 \%$ of each subgroup, the number of subjects in the reference population de- creased to 349 men and 357 women for Centaur hsTnl and to 352 men and 357 women for Atellica hsTnl, and the 99th percentile URLs significantly decreased to 13.5 and $13.4 \mathrm{ng} / \mathrm{L}$ for Centaur hsTnl and Atellica hsTnl, respectively.

When comparing men and women stratified according to age ( $\leq 55$ years vs. $>55$ years), the hsTnl 99th percentile URLs of Centaur hsTnl and Atellica hsTnl were significantly higher in men aged $>55$ years than in women aged $\leq 55$ years $[8,9]$ $(P<0.001$ for both; Fig. 4). The proportion of measurable samples above the limit of detection (LoD) was 91.5\% and 93.4\% for Centaur hsTnl and Atellica hsTnl, respectively (Table 2).
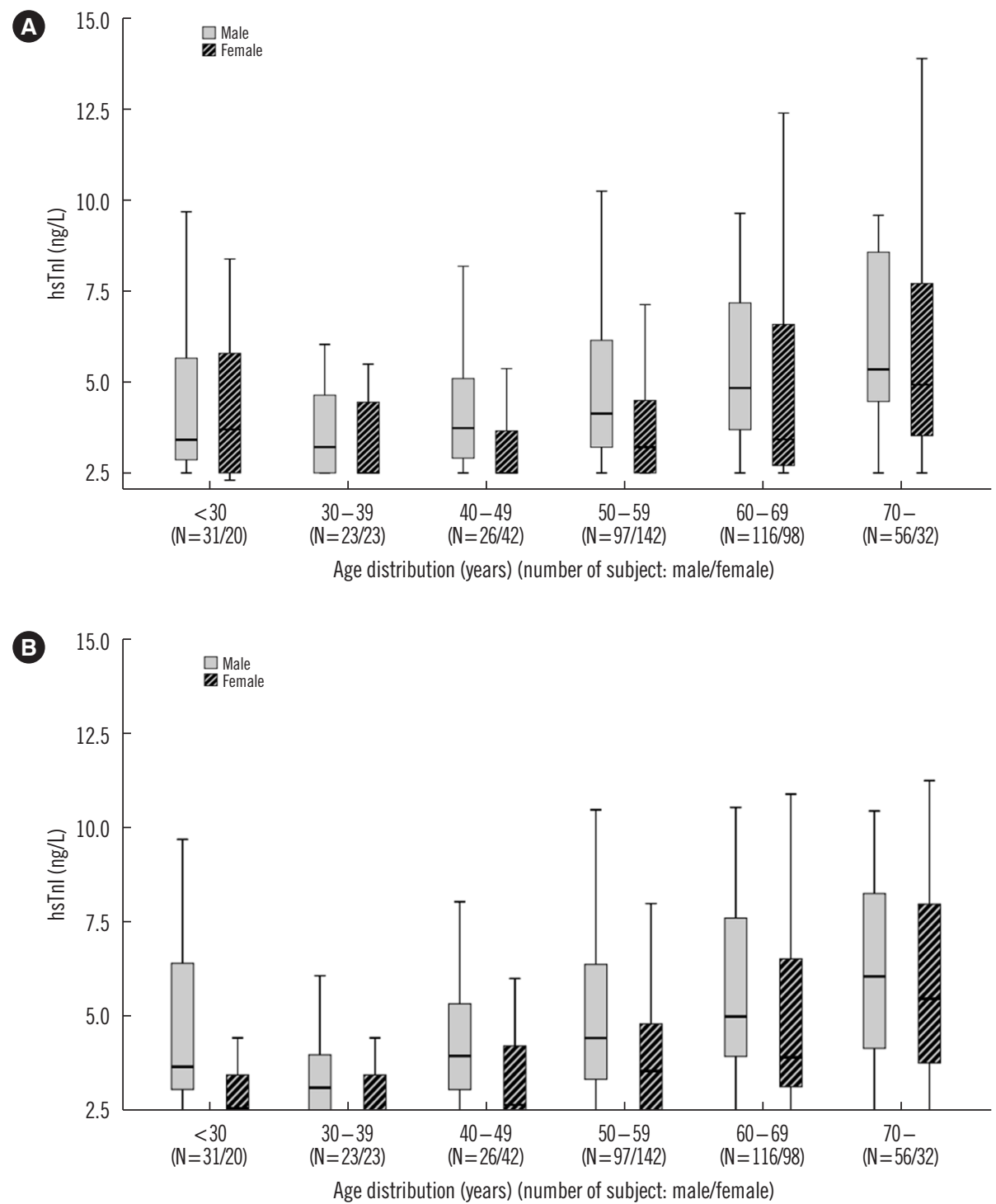

Fig. 3. Distribution of hsTnl values in men and women according to age and sex. The box indicates the 25th and 75th percentiles; the black line corresponds to the median value. (A) Centaur hsTnl, (B) Atellica hsTnl. Abbreviation: hsTnl, high-sensitivity troponin I. 


\section{DISCUSSION}

Various studies have indicated that Tnl 99th percentile URLs for Asians are lower than those for Caucasians [6, 7, 23-26]. Surprisingly, the 99th percentile URLs obtained in our study before outlier elimination were higher than those previously reported and those provided by the manufacturer (Siemens). Furthermore, they were higher than the 99th percentile URLs of 20.0 $\mathrm{ng} / \mathrm{L}$ (men) and $11.6 \mathrm{ng} / \mathrm{L}$ (women) for Siemens ADVIA Centaur
Tnl in Koreans [5, 6].

As shown in Figs. 3 and 4, the average hsTnl values increased with age, particularly at ages $\geq 55$ years. Additionally, hsTnl values were significantly higher in men than in women within the same age groups for both assays. The Mann-Whitney test for comparison of hsTnl values in men and women showed $P<0.031$ for both Centaur hsTnl and Atellica hsTnl. This result may be explained by the possibility of remaining outliers that were not eliminated during statistical analysis, especially in the
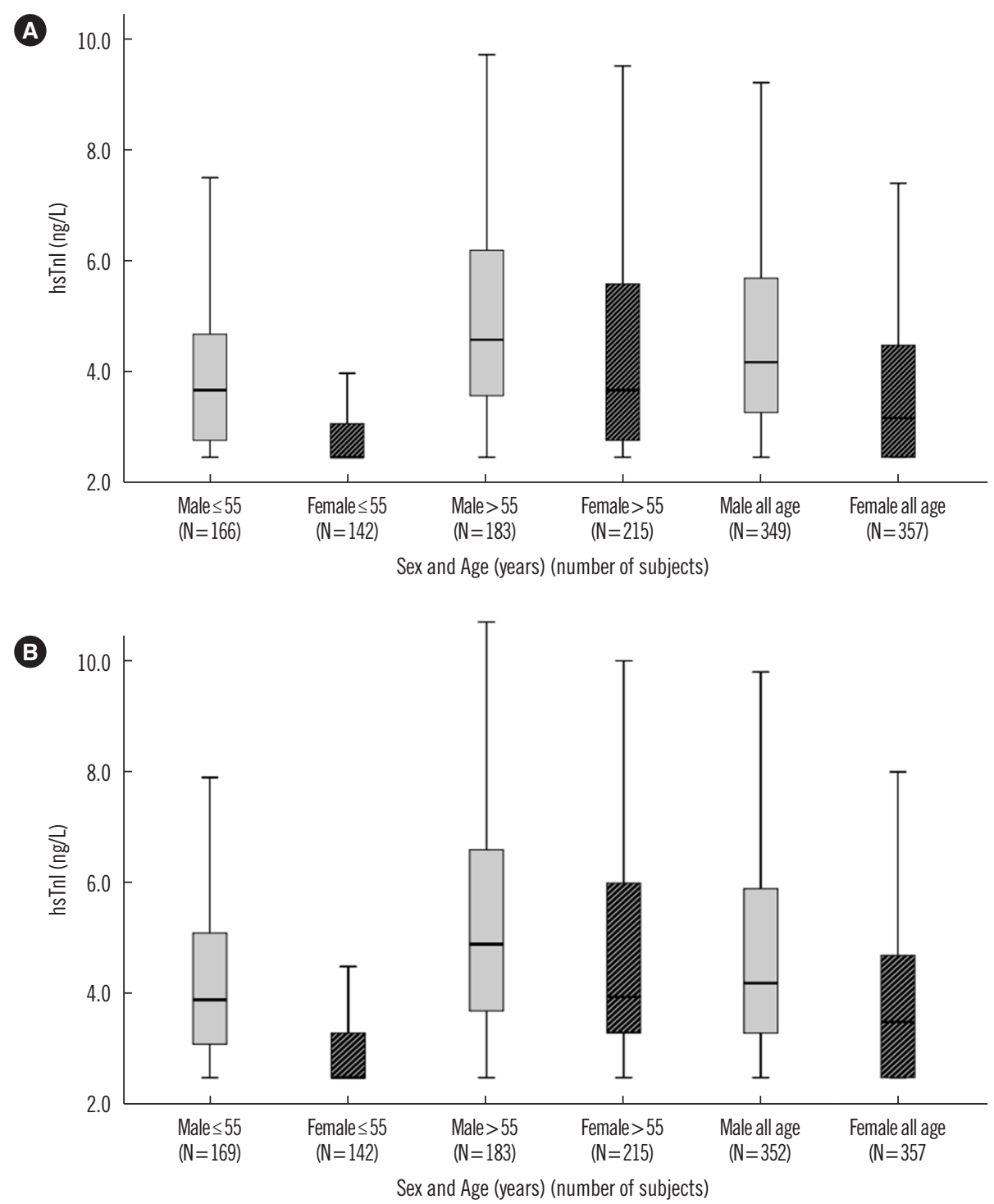

Fig. 4. hsTnl values in four subpopulations according to sex and age ( $\leq 55$ or $>55$ years). The box indicates the 25 th and 75 th percentiles, and the horizontal black line corresponds to the median value. The differences among the groups were statistically significant $(P<0.001$, Kruskal-Wallis test), with increasing tendencies in the following order: (A) Centaur hsTnl, $\leq 55$ years, women (2.5, 2.5-3.1), all ages, women (3.2, 2.5-4.5), $\leq 55$ years, men (3.7, 2.8-4.7), > 55 years, women (3.7, 2.8-5.6), all ages, men (4.2, 3.3-5.7), >55 years, men (4.6, 3.6-6.2) (B) Atellica hsTnl, $\leq 55$ years, women (2.5, 2.5-3.3), all ages, women (3.5, 2.5-4.7), $\leq 55$ years, men (3.9, 3.1-5.1), >55 years, women (4.0, 3.3-6.0), all ages, men (4.2, 3.3-5.9), and > 55 years, men (4.9, 3.7-6.6). Data are expressed as (median, IQR) ng/L. Abbreviations: hsTnl, high-sensitivity troponin I; IQR, interquartile range. 
Table 1. hsTnl 99th percentile URLs according to sex, age group, and before and after outlier elimination.

\begin{tabular}{|c|c|c|c|c|c|c|c|}
\hline Age group & Sex & $\mathrm{N}$ & $\begin{array}{l}\mathrm{N} \text { and } \% \text { of } \\
\text { subjects } \\
\text { eliminated }\end{array}$ & $\begin{array}{l}\text { Centaur hsTnl 99th } \\
\text { percentile URL }\end{array}$ & $\mathrm{N}$ & $\begin{array}{l}\mathrm{N} \text { and } \% \text { of } \\
\text { subjects } \\
\text { eliminated }\end{array}$ & $\begin{array}{l}\text { Atellica hsTnl 99th } \\
\text { percentile URL }\end{array}$ \\
\hline \multicolumn{8}{|c|}{ hsTnl 99th percentile URLs before outlier elimination } \\
\hline \multirow[t]{3}{*}{ All ages } & Male & 380 & N/A & $74.7(47.5-117.9)$ & 380 & $N / A$ & $75.2(47.3-122.7)$ \\
\hline & Female & 387 & $\mathrm{~N} / \mathrm{A}$ & $57.5(41.7-101.1)$ & 387 & $N / A$ & $55.1(38.2-95.8)$ \\
\hline & All & 767 & $\mathrm{~N} / \mathrm{A}$ & $60.4(445.7-94.8)$ & 767 & $N / A$ & $59.6(44.7-96.9)$ \\
\hline \multirow[t]{3}{*}{$\leq 55 \mathrm{yr}$} & Male & 182 & $\mathrm{~N} / \mathrm{A}$ & $114.5(65.9-256.1)$ & 182 & $N / A$ & $114.1(64.7-256.1)$ \\
\hline & Female & 158 & $\mathrm{~N} / \mathrm{A}$ & $64.8(52.1-71.1)$ & 158 & $N / A$ & $62.3(50.3-69.1)$ \\
\hline & All & 340 & $\mathrm{~N} / \mathrm{A}$ & $66.7(31.2-186.2)$ & 340 & $N / A$ & $66.1(30.3-185.9)$ \\
\hline \multirow[t]{3}{*}{$>55 \mathrm{yr}$} & Male & 198 & $\mathrm{~N} / \mathrm{A}$ & $72.6(54.0-116.7)$ & 198 & $N / A$ & $73.4(54.8-122.5)$ \\
\hline & Female & 229 & $\mathrm{~N} / \mathrm{A}$ & $97.1(53.6-187.6)$ & 229 & $N / A$ & $89.0(51.2-177.0)$ \\
\hline & All & 427 & $\mathrm{~N} / \mathrm{A}$ & $66.5(50.1-116.7)$ & 427 & $N / A$ & $67.0(49.9-122.7)$ \\
\hline \multicolumn{8}{|c|}{ hsTnl 99th percentile URLs after outlier elimination } \\
\hline \multirow[t]{3}{*}{ All ages } & Male & 349 & $31(8.2 \%)$ & $15.3(14.7-15.6)$ & 352 & $28(7.4 \%)$ & $15.5(15.5-16.0)$ \\
\hline & Female & 357 & $30(7.6 \%)$ & $11.9(11.1-12.4)$ & 357 & $30(7.6 \%)$ & $12.9(11.4-13.2)$ \\
\hline & All & 706 & $61(8.0 \%)$ & $13.5(12.8-13.6)$ & 709 & $58(7.6 \%)$ & $13.4(12.8-13.8)$ \\
\hline \multirow[t]{3}{*}{$\leq 55 \mathrm{yr}$} & Male & 166 & $16(8.6 \%)$ & $11.9(10.8-12.0)$ & 169 & $13(7.1 \%)$ & $11.8(10.7-12.4)$ \\
\hline & Female & 142 & $16(10.1 \%)$ & $7.0(6.8-7.0)$ & 142 & $16(9.5 \%)$ & $7.5(7.3-7.5)$ \\
\hline & All & 308 & 32 (9.4\%) & $10.3(9.6-10.5)$ & 311 & $28(8.2 \%)$ & $10.5(9.9-11.4)$ \\
\hline \multirow[t]{3}{*}{$>55 \mathrm{yr}$} & Male & 183 & 15 (7.6\%) & $16.9(16.4-18.6)$ & 183 & $15(7.6 \%)$ & $17.4(17.0-17.6)$ \\
\hline & Female & 215 & 14 (6.1\%) & $13.6(12.8-13.8)$ & 215 & $14(6.6 \%)$ & 13.6 (12.9-14.8) \\
\hline & All & 398 & $29(6.8 \%)$ & $16.2(14.8-16.5)$ & 397 & $30(7.0 \%)$ & $16.7(15.2-17.6)$ \\
\hline
\end{tabular}

Outliers were eliminated using a non-parametric (Tukey's 3-IQR) method as detailed in the Methods section. The values are presented as median (95\% CI) ng/L.

Abbreviations: URL, upper reference limit; hsTnl, high-sensitivity troponin I; IQR, interquartile range; $\mathrm{Cl}$, confidence interval; N/A, not applicable.

Table 2. hsTnl 99th percentile URLs (ng/L), CV\% at the URL, and proportion (\%) of measurable samples above the LoD for both assays

\begin{tabular}{lccccccc}
\hline \multirow{2}{*}{ Assay } & \multicolumn{3}{c}{ hsTnl 99th percentile URL (ng/L) } & \multirow{2}{*}{ LoD } & \multirow{2}{*}{ \% Measurable } & Assay designation \\
\cline { 2 - 4 } & Men (CV\%) & Women (CV\%) & All (CV\%) & (ng/L) & & \\
\hline Centaur hsTnl & $15.3(5.1 \%)$ & $11.9(5.3 \%)$ & $13.5(5.2 \%)$ & 2.2 & 91.5 & Level 3 (second generation, high sensitivity) \\
Atellica hsTnl & $15.5(3.4 \%)$ & $12.9(3.5 \%)$ & $13.4(3.5 \%)$ & 1.3 & 93.4 & Level 3 (second generation, high sensitivity)
\end{tabular}

URLs were determined based on AACC and AFCC guidelines. Assay designation was performed according to the proportion (\%) of measurable samples (Apple, 2009 [30]).

Abbreviations: URL, upper reference limit; LoD, limit of detection; AACC, American Association for Clinical Chemistry; IFCC, International Federation of Clinical Chemistry; hsTnl, high-sensitivity troponin I.

$>55$ years age group for both sexes.

Although the manufacturers did not provide detailed standards for the recruitment of the reference population in which 99th percentile URLs are to be determined, the proportion of subjects aged $\geq 50$ years for the 99th percentile URLs established by the manufacturer (Siemens) was $46 \%$. In our reference population, subjects aged $\geq 50$ years accounted for $78.7 \%$. The fact that the 99th percentile URLs in our study were lower than those provided by the manufacturer can be explained by the more stringent selection criteria used in our study than those used in the manufacturer's study.

The 99th percentile URLs in men and women were 15.3 and $11.9 \mathrm{ng} / \mathrm{L}$ and 15.5 and $12.9 \mathrm{ng} / \mathrm{L}$ for Centaur hsTnl and Atellica hsTnl, respectively. Kim, et al. [26] have reported that the 99th percentile URLs in men and women for Beckman Coulter Access hsTnl were 11.3 and $9.5 \mathrm{ng} / \mathrm{L}$, respectively, in the Ko- 
rean population. Apple, et al. [27] reported that hsTnl 99th percentile URLs were 35-43 ng/L for Centaur hsTnl and 30-43 $\mathrm{ng} / \mathrm{L}$ for Atellica, according to age in a United States population. In a mixed-sex population of 2,010 apparently healthy individuals from Italy, the 99th percentile URL value for Centaur hsTnl was $47.30 \mathrm{ng} / \mathrm{L}$ [28]. Based on the results of these and our studies, we conclude that hsTnl 99th percentile URLs are higher for Caucasians than for East Asians and specifically, Koreans.

The hsTnl 99th percentile URLs are significantly higher in men than in women across all ages [23, 29]. In our previous study, 99th percentile URLs calculated for Centaur Tnl in Korean men and women were 20.0 and $11.6 \mathrm{ng} / \mathrm{L}$, respectively [6]. As hsTnl 99th percentile URLs are higher in men than in women, it is recommended that different hsTnl 99th percentile URLs are used for men and women. Furthermore, 99th percentile URLs are higher in the older population than in the younger population. Thus, establishing guidelines for age distribution in the reference population used for calculating hsTnl 99th percentile URLS should be considered.

We previously reported a CV of $43.0 \%$ at the 99th percentile URL using Centaur Tnl, the predecessor of Centaur hsTnl [6]. In contrast, in this study, Centaur hsTnl and Atellica hsTnl showed CVs of $5.2 \%$ and $3.5 \%$, respectively, at the 99th percentile URLs, indicating an improvement in precision. Applying the criteria suggested by Apple [30], the CVs at the 99th percentile URLs for Centaur hsTnl and Atellica hsTnl were $\leq 10 \%$, which is clinically considered "Guideline acceptable." The measurable normal values (\%), defined as the proportions of specimens with values above the LoD, were $91.5 \%$ and $93.4 \%$ for Centaur hsTnl and Atellica hsTnl, respectively. Both systems were classified as level 3 (second generation, high sensitivity) in a scorecard designation [30]. Current AACC and IFCC guidelines state that hsTn assays must have an analytical imprecision of $<10 \% \mathrm{CV}$ at the 99th percentile URL in a healthy population and should be able to measure CTn above the LoD in $50 \%$ of the healthy population, ideally 95\%. Both hsTnl assays can be defined as highly sensitive according to the AACC and IFCC criteria [11].

We performed the NT-proBNP assay in the reference population to screen for subjects with asymptomatic cardiac diseases, which can be considered a strength of the current study. However, we are aware that NT-proBNP assays cannot screen cardiac patients perfectly.

This study had some limitations. First, the reference population used to determine hsTnl 99th percentile URLs was smaller than those used in previous similar studies [8, 9]. The number of outliers was relatively high, which reduced the size of the final reference population used to calculate the 99th percentile URLs. It was difficult to include subjects in the reference population as our study was a single-center study, as opposed to multicenter or multinational studies. Second, in the comparison study, we did not use samples covering a wide range of values. In the comparison between Centaur Tnl and Centaur hsTnl, samples with values ranging from $0.0 \mathrm{ng} / \mathrm{L}$ to $23.5 \mathrm{ng} / \mathrm{L}$ were used, which did not cover the entire analytical measurement range of the assays. In comparing NT-proBNP values between the Cobas C8000 and Atellica IM systems, there was a gap between 4,100 ng/L and 15,700 ng/L, and thus, our results do not reflect actual assay results obtained in clinical laboratories. Third, we used frozen serum instead of fresh serum for analysis, which may have influenced the study results. Finally, while recruiting the reference population, we did not use imaging modalities, such as cardiac sonography or electrocardiography. Asymptomatic cardiac patients may have been missed, which may have increased the 99th percentile URLS.

Currently, hsTnl and hsTnT values are critical in diagnosing cardiac diseases, especially, acute myocardial infarction. These values are interpreted using various methods, and quality control of hsTnl and hsTnT is becoming essential to obtain accurate hsTnl and hsTnT values [31, 32]. Our study highlighted the importance of outlier elimination in determining precise 99th percentile URL values.

Considering the clinical significance of hsTnl 99th percentile URLs, determining 99th percentile URLs using a strictly selected population and a reliable and standardized method, including proper outlier elimination, is important. To the best of our knowledge, this is the first study to report an outlier elimination effect in calculating hsTnl 99th percentile URL values, especially in a Korean population. When introducing a new hsTnl assay system, the 99th percentile URLs should be recalculated for clinical application as the Centaur hsTnl assay was not harmonized with the Centaur Tnl system according to our results.

\section{ACKNOWLEDGMENTS}

We thank Editage (www.editage.co.kr) for editing and reviewing the English language translation of this manuscript. The authors also wish to thank Young Kim and Siemens Healthineers for supporting this project and providing technical assistance. 


\section{AUTHOR CONTRIBUTIONS}

Ahn S performed the experiments and wrote the paper; Kim HK collected the samples; Lee W revised the paper; Min WK and Chun S designed the study. All authors have accepted responsibility for the entire content of the manuscript and have approved its submission.

\section{CONFLICT OF INTERESTS}

None declared.

\section{RESEARCH FUNDING}

None declared.

\section{ORCID}

Sunyoung Ahn

Hyun-Ki Kim

Woochang Lee

Sail Chun

Won-Ki Min https://orcid.org/0000-0003-3892-0921

https://orcid.org/0000-0002-3299-5298

https://orcid.org/0000-0003-3956-6397

https://orcid.org/0000-0002-5792-973X

https://orcid.org/0000-0002-5158-2130

\section{REFERENCES}

1. Bodor GS, Porterfield D, Voss EM, Smith S, Apple FS. Cardiac troponinI is not expressed in fetal and healthy or diseased adult human skeletal muscle tissue. Clin Chem 1995;41:1710-5.

2. Thygesen K, Alpert JS, Jaffe AS, Chaitman BR, Bax JJ, Morrow DA, et al. Fourth universal definition of myocardial infarction (2018). Circulation 2018;138:e618-51.

3. Reichlin T, Hochholzer W, Bassetti S, Steuer S, Stelzig C, Hartwiger S, et al. Early diagnosis of myocardial infarction with sensitive cardiac troponin assays. N Engl J Med 2009;361:858-67.

4. Mahajan VS and Jarolim P. How to interpret elevated cardiac troponin levels. Circulation 2011;124:2350-4

5. Collinson PO, Heung YM, Gaze D, Boa F, Senior R, Christenson R, et al. Influence of population selection on the 99th percentile reference value for cardiac troponin assays. Clin Chem 2012;58:219-25.

6. Ko DH, Jeong TD, Cho EJ, Lim J, Ji M, Lee K, et al. The 99th percentile values of six cardiac troponin assays established for a reference population using strict selection criteria. Clin Chim Acta 2017;464:1-5.

7. Di Pietro M, Dipalo M, Rocchi MBL, Musa R, Avanzini P, Gnocchi C, et al. Assessment of Access hsTnl 99th percentiles upper reference limits following IFCC recommendations. Clin Chim Acta 2019;492:26-8.

8. Clerico A, Zaninotto M, Ripoli A, Masotti S, Prontera C, Passino C, et al. The 99th percentile of reference population for CTnl and CTnT assay: methodology, pathophysiology and clinical implications. Clin Chem Lab Med 2017;55:1634-51.

9. Clerico A, Ripoli A, Zaninotto M, Masotti S, Musetti V, Ciaccio M, et al. Head-to-head comparison of plasma cTnl concentration values mea- sured with three high-sensitivity methods in a large Italian population of healthy volunteers and patients admitted to emergency department with acute coronary syndrome: A multi-center study. Clin Chim Acta 2019; 496:25-34.

10. Sandoval $Y$ and Apple FS. The global need to define normality: the 99th percentile value of cardiac troponin. Clin Chem 2014;60:455-62.

11. Wu AHB, Christenson RH, Greene DN, Jaffe AS, Kavsak PA, OrdonezLlanos J, et al. Clinical Laboratory practice recommendations for the use of cardiac troponin in acute coronary syndrome: expert opinion from the Academy of the American Association for Clinical Chemistry and the Task Force on Clinical Applications of Cardiac Bio-markers of the International Federation of Clinical Chemistry and Laboratory Medicine. Clin Chem 2018;64:645-55.

12. Hickman PE, Koerbin G, Potter JM, Abhayaratna WP. Statistical considerations for determining high-sensitivity cardiac troponin reference intervals. Clin Biochem 2017;50:502-5.

13. Eggers KM, Apple FS, Lind L, Lindahl B. The applied statistical approach highly influences the 99th percentile of cardiac troponin I. Clin Biochem 2016;49:1109-12.

14. Clerico A, Zaninotto M, Passino C, Aspromonte N, Piepoli MF, Migliard $\mathrm{M}$, et al. Evidence on clinical relevance of cardiovascular risk evaluation in the general population using cardio-specific biomarkers. Clin Chem Lab Med 2020;59:79-90.

15. Farmakis D, Mueller C, Apple FS. High-sensitivity cardiac troponin assays for cardiovascular risk stratification in the general population. Eur Heart J 2020;41:4050-6.

16. CLSI. Evaluation of precision performance of quantitative measurement methods: approved guideline. 2nd ed EP5-A2. Wayne, PA: Clinical and Laboratory Standards Institute, 2004.

17. CLSI. Evaluation of the linearity of quantitative measurement procedures: A statistical approach: approved guideline. EP6-A. Wayne, PA: Clinical and Laboratory Standards Institute, 2003.

18. Ricos C, Alvarez V, Cava F, Garcia-Lario JV, Hernandez A, Jimenez CV, et al. Desirable Biological Variation Database specifications: Desirable specifications for total error, imprecision, and bias, derived from intraand inter-individual biologic variation. https://www.westgard.com/biodatabase1.htm (Updated in 2014).

19. CLSI. Method comparison and bias estimation using patient samples: approved guidelines. 2nd ed. EP9-A2. Wayne, PA: Clinical and Laboratory Standards Institute, 2002.

20. Franzini M, Lorenzoni V, Masotti S, Prontera C, Chiappino D, Latta DD, et al. The calculation of the cardiac troponin T 99th percentile of the reference population is affected by age, gender, and population selection: a multicenter study in Italy. Clin Chim Acta 2015;438:376-81.

21. Hickman PE, Potter JM, Aroney C, Koerbin G, Southcott E, Wu AH, et al. Cardiac troponin may be released by ischemia alone, without necrosis. Clin Chim Acta 2010;411:318-23.

22. Tukey J. Exploratory data analysis, 1977. http://www.ru.ac.bd/wp-content/uploads/sites/25/2019/03/102_05_01_Tukey-Exploratory-DataAnalysis-1977.pdf

23. Clerico A, Masotti S, Musetti V, Ripoli A, Aloe R, Di Pietro M, et al. Evaluation of 99th percentile and reference change values of the hs-cTnl method using ADVIA Centaur XPT platform: A multicenter study. Clin Chim Acta 2019;495:161-6.

24. Ji M, Moon HW, Hur M, Yun YM. Determination of high-sensitivity cardiac troponin I 99th percentile upper reference limits in a healthy Korean population. Clin Biochem 2016;49:756-61.

25. Moon SY, Kim N, Lee SM, Yu S, Jun KR, Kim HH. 99th percentile upper reference limit of AccuTnI+3 in a Korean reference population: a multicenter study using fresh serum. Clin Chem Lab Med 2019;57: 


\section{Ahn S, et al.}

e282-4.

26. Kim S, Yoo SJ, Kim J. Evaluation of the new Beckman Coulter Access hsTnl: 99th percentile upper reference limits according to age and sex in the Korean population. Clin Biochem 2020;79:48-53.

27. Apple FS, Wu AHB, Sandoval Y, Sexter A, Love SA, Myers G, et al. SexSpecific 99th percentile upper reference limits for high sensitivity cardiac troponin assays derived using a universal sample bank. Clin Chem 2020;66:434-44.

28. Musetti V, Masotti S, Prontera C, Storti S, Ndreu R, Zucchelli GC, et al. Evaluation of the analytical performance of a new ADVIA immunoassay using the Centaur XPT platform system for the measurement of cardiac troponin I. Clin Chem Lab Med 2018;56:e229-31.
29. Petersmann A, Ittermann T, Fries C, Lubenow N, Kohlmann T, Kallner A, et al. Comparison of the 99th percentiles of three troponin I assays in a large reference population. Clin Chem Lab Med 2013;51:2181-6.

30. Apple FS. A new season for cardiac troponin assays: it's time to keep a scorecard. Clin Chem 2009;55:1303-6.

31. Kim JW, Kim H, Yun YM, Lee KR, Kim HJ. Absolute change in highsensitivity cardiac troponin I at three hours after presentation is useful for diagnosing acute myocardial infarction in the emergency department. Ann Lab Med 2020;40:474-80.

32. Li T, Cao S, Wang Y, Xiong Y, He Y, Ke P, et al. Moving rate of positive patient results as a quality control tool for high-sensitivity cardiac troponin T assays. Ann Lab Med 2021;41:51-9. 
Supplemental Data Table S1. Precision profiles of Siemens Atellica IM BNP, Atellica IM NT-proBNP, Centaur hsTnl, and Atellica hsTnl

\begin{tabular}{|c|c|c|c|c|c|c|c|}
\hline \multirow{2}{*}{ Analyte (Instrument) } & \multirow{2}{*}{ Unit } & \multirow{2}{*}{ Value } & \multirow{2}{*}{ Mean } & \multicolumn{4}{|c|}{ CV (\%) } \\
\hline & & & & Within-run & Between-run & Between-day & Total \\
\hline \multirow[t]{3}{*}{ BNP (Atellica) } & $\mathrm{ng} / \mathrm{L}$ & 1 & 86.37 & 2.0 & 1.6 & 1.6 & 3.0 \\
\hline & & 2 & 344.19 & 2.1 & 1.7 & 1.8 & 3.2 \\
\hline & & 3 & $1,239.91$ & 1.9 & 1.7 & 1.5 & 3.0 \\
\hline \multirow[t]{3}{*}{ NT-proBNP (Atellica) } & $\mathrm{ng} / \mathrm{L}$ & 1 & 168.14 & 1.9 & 2.6 & 0.0 & 3.2 \\
\hline & & 2 & 518.69 & 1.7 & 2.6 & 0.0 & 3.1 \\
\hline & & 3 & $5,764.03$ & 1.8 & 1.8 & 1.0 & 2.7 \\
\hline \multirow[t]{3}{*}{ hsTnl (Centaur) } & $\mathrm{ng} / \mathrm{L}$ & 1 & 34.57 & 2.0 & 3.4 & 1.0 & 4.1 \\
\hline & & 2 & $4,553.27$ & 1.7 & 0.1 & 1.4 & 2.2 \\
\hline & & 3 & $11,887.13$ & 1.6 & 1.1 & 1.3 & 2.3 \\
\hline \multirow[t]{3}{*}{ hsTnl (Atellica) } & $\mathrm{ng} / \mathrm{L}$ & 1 & 38.32 & 2.8 & 1.7 & 2.6 & 4.1 \\
\hline & & 2 & $4,517.89$ & 1.5 & 1.4 & 1.0 & 2.3 \\
\hline & & 3 & $11,804.30$ & 1.3 & 1.2 & 1.0 & 2.0 \\
\hline
\end{tabular}

Abbreviations: BNP, B-type natriuretic peptide; NT-proBNP, N-terminal pro-B-type natriuretic peptide; hsTnl, high-sensitivity troponin I. 
Ahn S, et al.

The 99th percentile URL of hsTnl assays
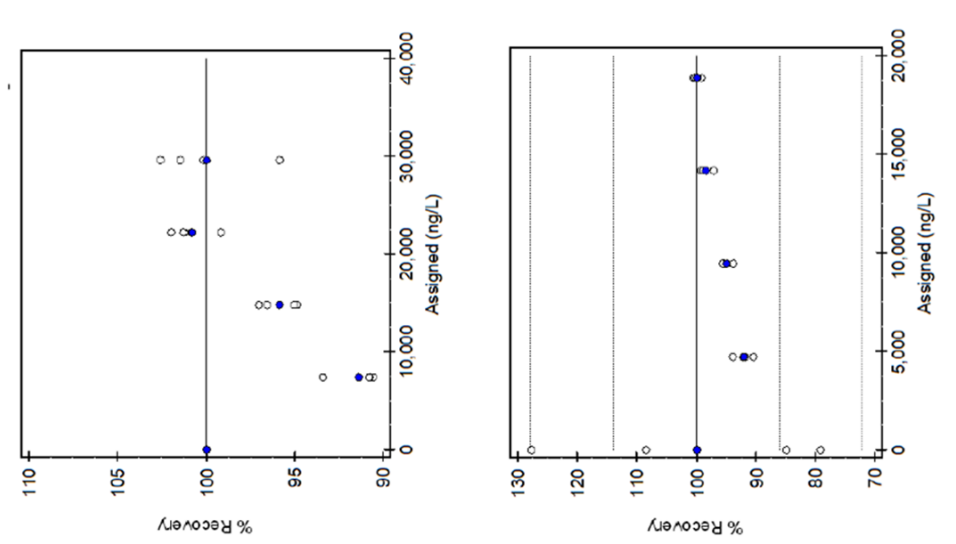

(1)
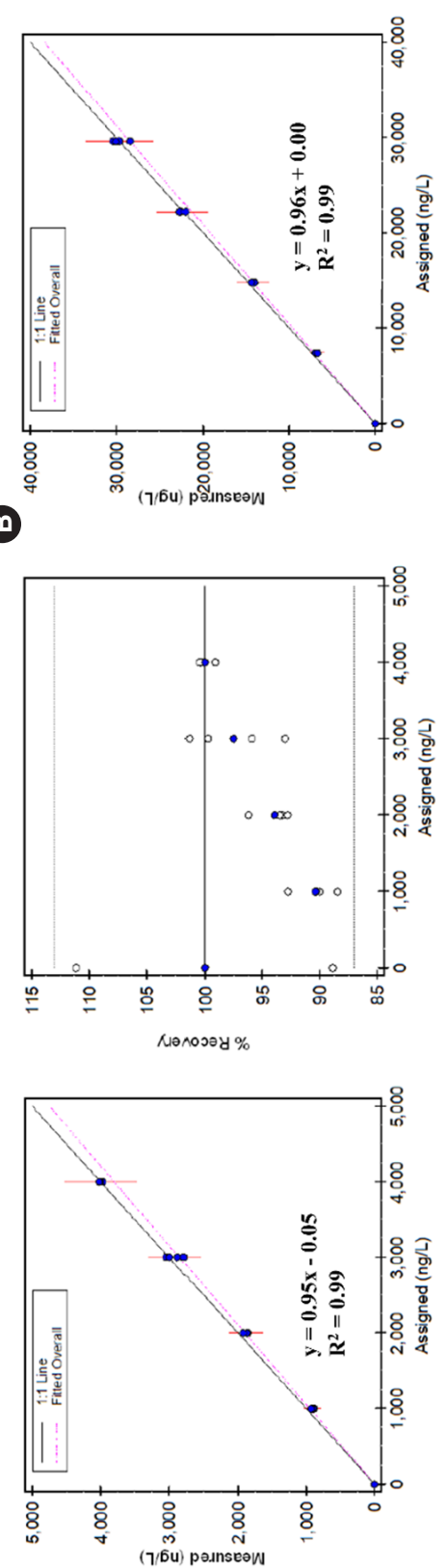

(อ)
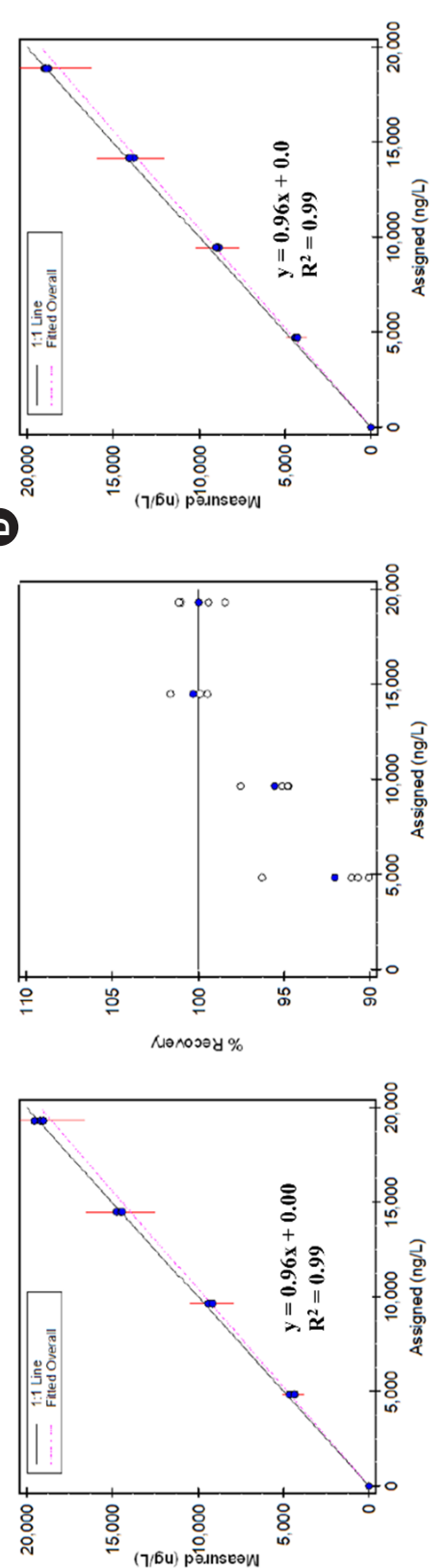

(

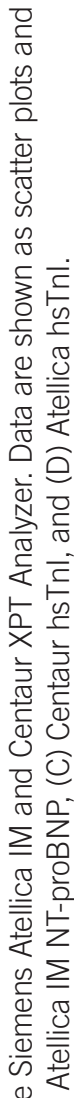

$\stackrel{\oplus}{\mp}$

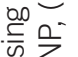

ज证

य)

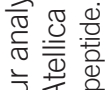

으 $\frac{1}{2}$

으 $\$$

రृ

就 䨌

宁

号 造

过 on

啐纷

约

त)

곤

힌을 음

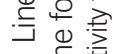

도

ज ज

aํํ은

it 든

ॠ 응 ত্য 닐

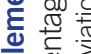



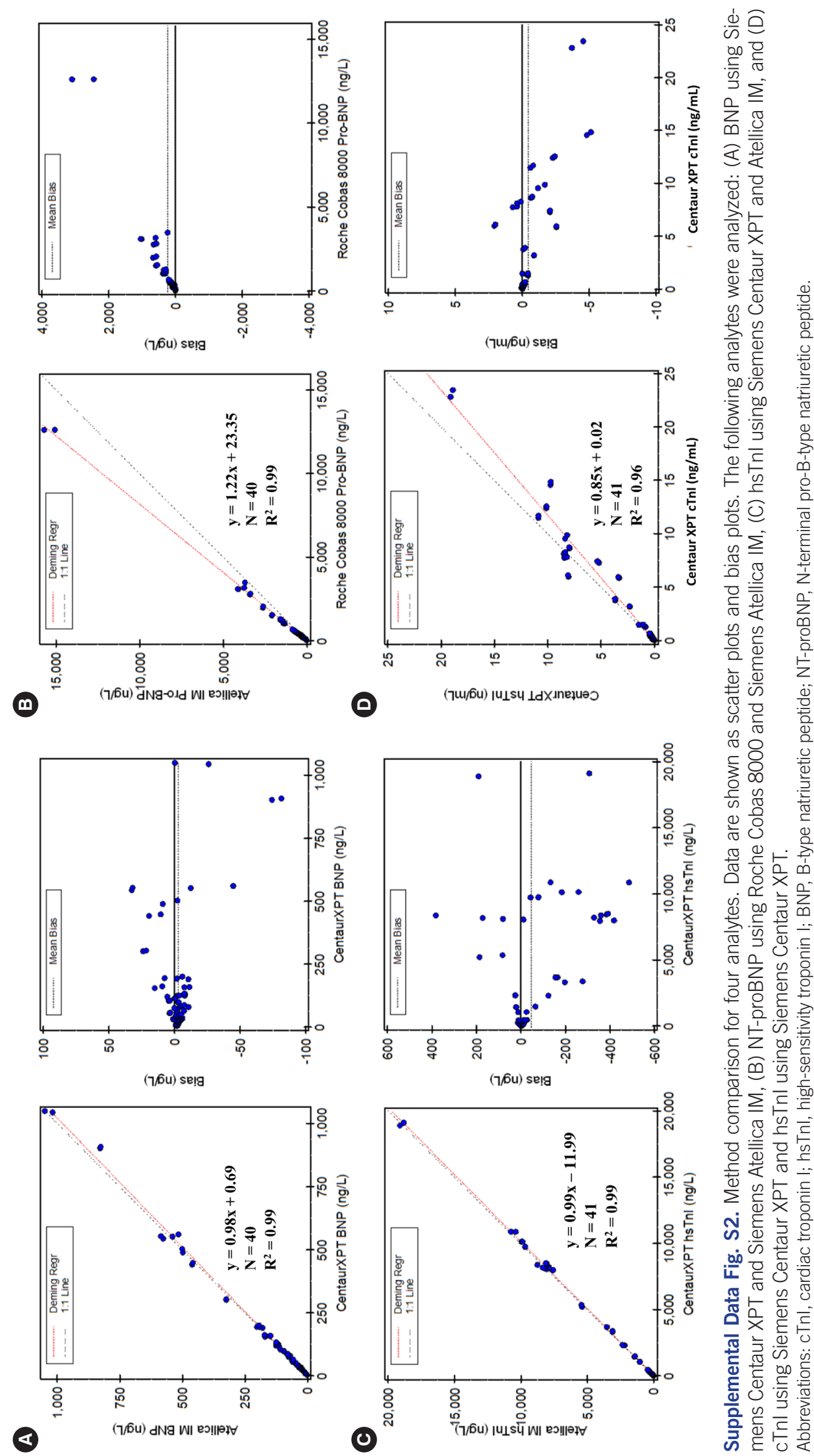

कि 